\title{
Influence of channel dredging on subsea shield tunnel using FEM
}

\author{
Xianghong Chen ${ }^{1,}$, Lianjin Tao ${ }^{1}$ and Xi Chen ${ }^{2, b}$ \\ ${ }^{1}$ Beijing University of Technology, China College Of Civil Engineering Beijing 100124, P.R.China \\ ${ }^{2}$ Zhong Zi Tai Ke Traffic Construction Technology Co.,Ltd,China, 100083, P.R.China \\ a06115214@bjtu.edu.cn, b840384890@qq.com
}

Keywords: Channel dredging; underwater tunnel; back silting; finite element method.

Abstract. Subsea tunnel construction has become the prevalent tendency connecting countries and districts, Controlling and minimizing the negative influence of channel dredging on tunnel have great significance throughout the tunnel construction and operation phase. So a three-dimensional finite element model of soil-tunnel and port structures was established given the tunnel surrounding rock to be isotropic elasto-plastic media meeting Drucker-Prager criterion of continuum, the construction conditions of channel excavation, back silting and desilting were investigated and the calculation results were analyzed. The numerical calculation results show that: the channel dredging increased the deformation and stress discrepancy along the tunnel longitudinal direction, the distinct affection happened at the locations beneath the ocean bottom, and the maximum value occurred mainly at the position of tunnel segments in the domain of retaining wall. Moreover, channel dredging decreased the ground settlement induced by tunnel excavation, and siltation made partially the spring-back displacement. Therefore an evaluation of the influence of repeated loads on tunnel should also be considered integrally to determine the dredging scheme in the future project.

\section{Introduction}

Normalized channel dredging undertakes the task of ensuring navigation and maintaining the ecological environment of waterway in various ports [1]; the principal components of dredging are excavation, transport and disposal of earth material. Undersea dredging excavation becomes very challenging when encountering nearby buildings, and is easy to cause several unwanted effects, such as ground vibration, displacement and stress having enough potential to cause damage to the surrounding structures and environment [2 6]. Though all these effects can't be completely eliminated using improved construction method, they can be minimized to acceptable levels to avoid damage through investigated and designed systematically. Over the past several years, most undersea shield tunnel appeared in China, such as Xiamen Xiang' an subsea tunnel, Qingdao Kiaochow bay subsea tunnel, and Dalian bay tunnel, etc [7,8], Based on the previous research conclusions, a numerical analysis method was applied to model the effect of channel dredging on proposed Mom bay subsea tunnel. The details of these studies are illustrated in the paper.

\section{Description of the construction project}

Proposed Mom bay subsea tunnel is designed to connect Mawan port and Dachan bay port in Shenzhen, with a view to meet the increased traffic demand and to facilitate land transportation. The project will involve construction of circular tunnel with $15 \mathrm{~m}$ outer diameter, $0.65 \mathrm{~m}$ thickness, $2 \mathrm{~m}$ width and $15 \mathrm{~m}$ spacing distance, the embedded depth is $10 \mathrm{~m}$ from channel bottom after dredging, and the mean dredging depth is $12 \mathrm{~m}$, the cross section size of port retaining wall is $20 \mathrm{~m} \times 4 \mathrm{~m}$ above water table. Fig. 1 shows the location of tunnel and port around the dredging site.

The drilling exposure of geological conditions from top to bottom mainly exposed: artificial filled soil and filled rock $\left(Q^{\mathrm{ml}}\right)$ back of the retaining wall on shore, silt layer $\left(\mathrm{Q}^{\mathrm{ml}}\right)$ under ocean bottom, clay, gravel, medium and coarse sand $\left(\mathrm{Q}^{\mathrm{al}+\mathrm{pl}}\right)$, strongly $\sim$ slightly weathered granite $\left(\mathrm{J}_{\mathrm{x}}-\mathrm{Qb}\right)$ and deep tectonic rock (f). 

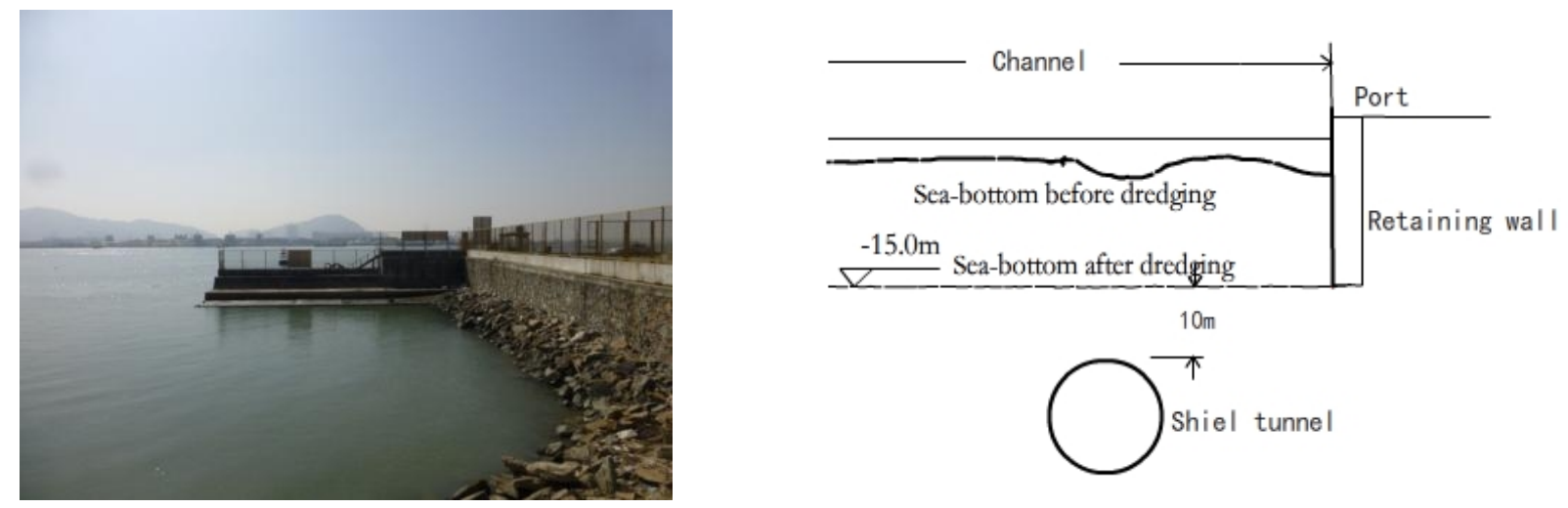

Fig.1 Locations of structures around the dredging site

\section{Finite element model with plastic deformation elements}

Rgeological Model. a Drucker-Prager model with no hardening considered here is used to simulate the geological properties, the material parameters are presented in Table 1, the relationship between stress tensor increment $d \sigma$ and strain increment $d e$ can then be expressed as [9]:

$$
\left.\begin{array}{c}
d \sigma=C:\left(d e-d e^{p}\right) \\
d e^{p}=d \xi \frac{\partial f}{\partial \sigma}
\end{array}\right\}
$$

where $C=\frac{E \mu}{(1-2 \mu)(1+\mu)} \delta_{i j} \otimes \delta_{i j}+\frac{E}{2(1+\mu)}\left(\delta_{i l} \delta_{j k}+\delta_{i k} \delta_{j l}\right)$ denotes the tensor of linear isotropic elasticity, $d e$ and $d e^{p}$ is the total and plastic strain, $E$ and $\mu$ is the Young's modulus and the poisson's ratio respectively, $d \xi$ is the plastic multiplier, $f$ is the yield function ie:

$f(\sigma)=\alpha J_{1}+\sqrt{J_{2}}-k$

$J_{1}$ is the first invariant of the stress tensor, and $J_{2}$ is the second invariant of the stress deviator tensor. $\alpha$ and $k$ denote the material constants, which can be expressed as surrounding rock's cohesive force $c$ and friction angle $\varphi$ :

$$
\left.\begin{array}{c}
\alpha=\frac{2 \sin \varphi}{\sqrt{3}(3 \pm \sin \varphi)} \\
k=\frac{6 c \cos \varphi}{\sqrt{3}(3 \pm \sin \varphi)}
\end{array}\right\}
$$

Table 1 The parameters of materials

\begin{tabular}{cccccc}
\hline Materials & $E / \mathrm{GPa}$ & $\mu$ & $\gamma /\left(\mathrm{kN} \cdot \mathrm{m}^{-3}\right)$ & $c / \mathrm{kPa}$ & $\varphi /\left({ }^{\circ}\right)$ \\
\hline Tunnel surporting & 34.50 & 0.20 & 25 & - & - \\
Retaining wall & 30.00 & 0.20 & 23 & - & - \\
Grounting reinforced soil & 0.20 & 0.28 & 22 & 400 & 30 \\
Filled soil and rock & 0.02 & 0.30 & 19 & 20 & 20 \\
Medium and coarse sand & 0.02 & 0.35 & 20 & 20 & 20 \\
Strongly weathered granite & 0.12 & 0.32 & 22 & 300 & 28 \\
Sligthtly weathered granite & 4.50 & 0.25 & 25 & 6000 & 35 \\
\hline
\end{tabular}

Finite Element Model. Numerical simulations for the subsea shield tunnel have been carried out with the finite element software ANSYS [10], based on geometrical modeling, three dimensional meshes have been adopted for the tetrahedral, hexahedral and pyramidal elements. Fig.2(a) shows the 1/4 soil-tunnel mesh modeling, in the simulations, the length of Marine and terrestrial area is $60 \mathrm{~m}$, the distance between the boundary of the surrounding soil and the tunnel contour on the same side is more than 2.5 times the diameter of this shield tunnel, the degrees of freedom in the coordinate direction have been constrained at the border nodes. The final three-dimensional size of this soil-tunnel system model is $140 \mathrm{~m} \times 100 \mathrm{~m} \times 70 \mathrm{~m}$. The number of nodes and elements reaches 73140 and 68450 , respectively. 
As shown in Fig.2(b), three points at the tunnel section along the tunnel longitudinal direction are chosen to evaluate the degree of deformation and principal stress, the distance of point 1 from the boundary of the sea area is $20 \mathrm{~m}$, and the distance of point 3 from the boundary of the land area is $20 \mathrm{~m}$ too, and point 2 is chosen at the mid-position of tunnel.
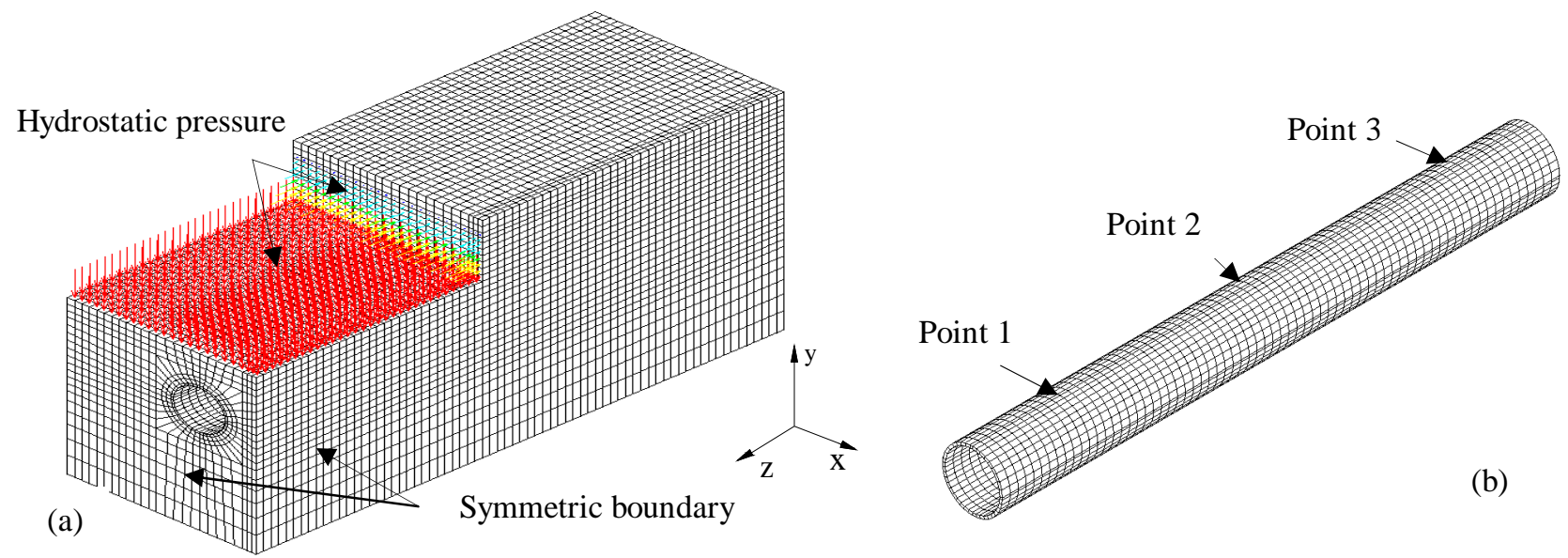

Fig.2 Finite element model (a); and measuring positions (b)

The whole construction involves four construction steps: the shield excavation, planning channel dredging, siltation and the 2th desilting are shown in Table 2, the designed back silting thickness is $2 \mathrm{~m}$, and the grouting pressure is $0.3 \mathrm{Mpa}$ on tunnel lining and $0.15 \mathrm{Mpa}$ at tunnel face. The life and death technique will be used to realize the simulation of the process of tunnel excavation, and the Newton-Raphson iterative method will be used to solve the construction process.

Table 2 Illustrations of construction steps

\begin{tabular}{ccccc}
\hline stages & $\begin{array}{c}\text { Shield tunenl } \\
\text { excavation }\end{array}$ & $\begin{array}{c}\text { Planning channel } \\
\text { dredging }\end{array}$ & $\begin{array}{c}\text { Siltation to } \\
2 \mathrm{~m}\end{array}$ & $\begin{array}{c}\text { The 2th } \\
\text { dredging }\end{array}$ \\
\hline $\begin{array}{c}\text { Construction } \\
\text { steps }\end{array}$ & $1-70$ & $71-77$ & 78 & 79 \\
\hline
\end{tabular}

\section{Analysis of calculation results}

Final calculations have produced the response of the subsea shield tunnel under channel dredging. Fig. 3 and Fig. 4 show the maximum displacement and principal stress distribution in the tunnel segments along the longitudinal direction. Such graphs provide a direct and global understanding of the tunnel segments' deformation and stress under varied construction steps.

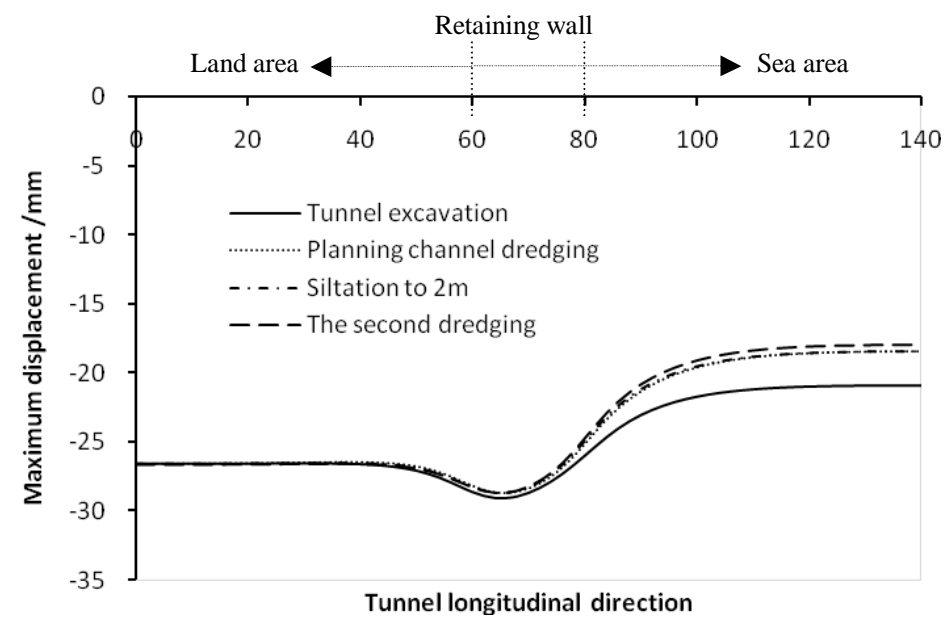

Fig. 3 Displacement distribution in the tunnel segments along the longitudinal direction 

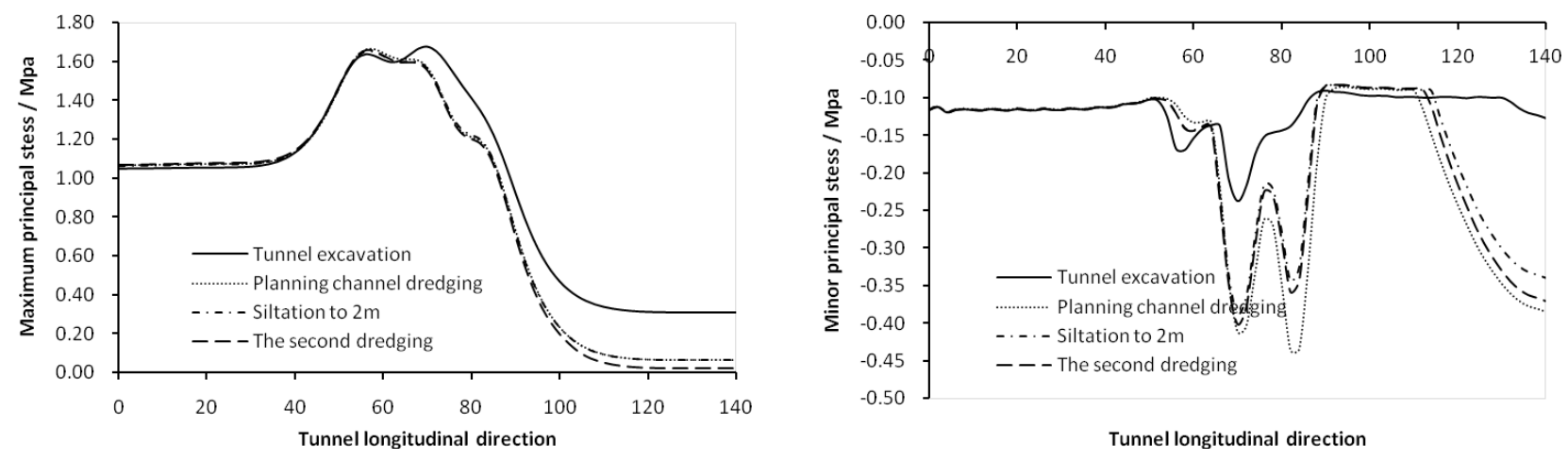

Fig.4 Maximum and minor principal stress in the tunnel segments along the longitudinal direction

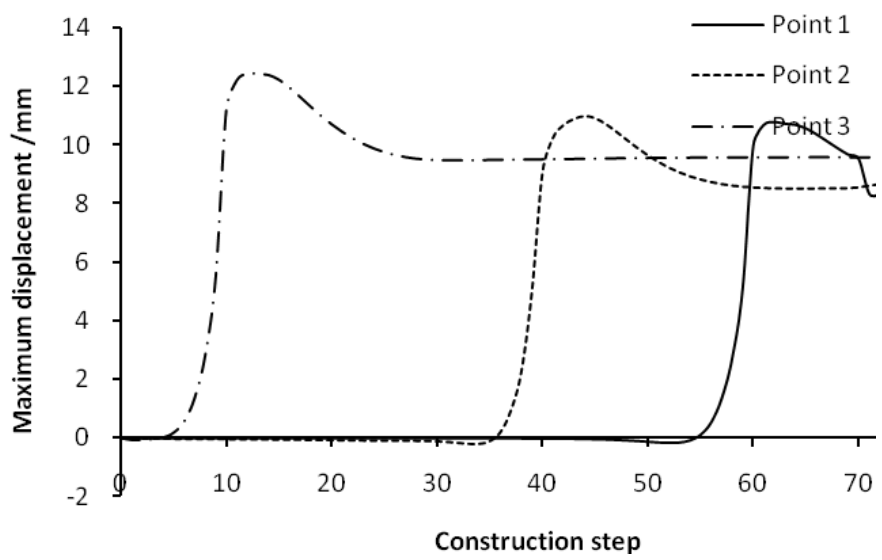

Fig.5 Displacement distributions of measuring points under construction steps
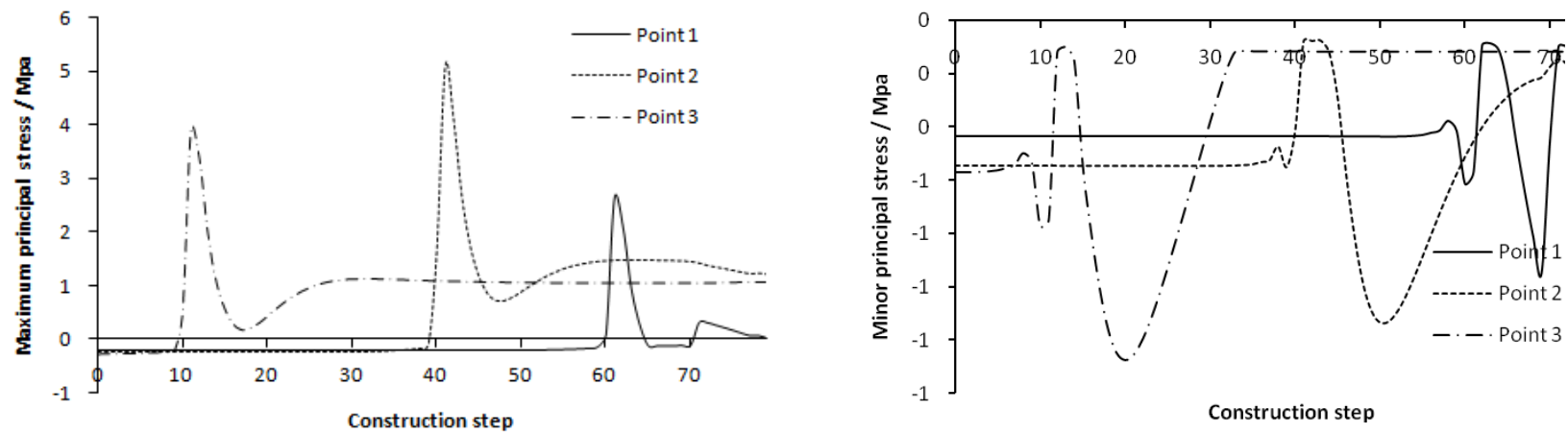

Fig.6 Maximum and minor principal stress distributions of measuring points under construction steps

Fig. 5 and Fig. 6 show the variation in maximum displacement and principal stress at the positions of Point 1, Point 2 and Point 3 under construction steps. By comparison, it is found that, the resilience curves influenced by channel dredging at Point 1 and Point 2 are more distinct than Point 3. Furthermore, in the domain of retaining wall, the maximum stress of tunnel is higher than that in land or sea area, Most importantly, the joint section between tunnel and shaft exists at the nearby position, from the point of view of avoiding faulted gaps and excessive relative displacement beyond threshold between shield tunnel segments, special strengthening measures should be brought in the design of tunnel segments at the junction of land and sea accompanying ground treatment.

Owing to space reasons, the effects of channel dredging considering the joint types between tunnel segments, between the tunnel and shaft will be studied afterwards as well as the stability and fatigue damage analysis of structure in the future. 


\section{Conclusions}

In the context, the finite element method is introduced to analyze the influence of channel dredging on subsea tunnel, and the calculation results can be shown as following by established programming models with discrete elements considering the nonlinear behavior of geological conditions:

1) The uplifted displacement of structure beneath the ocean bottom induced by tunnel excavation and dredging can counteract partially the settlement due to the ground gravity.

2) The maximum differential settlement and stress occurred at the tunnel segments in the positions connecting land and sea by numerical simulation.

3) The effect of channel dredging on tunnel segments at the positions beneath sea bottom is more distinct than that in the land region.

4) The study of tunnel segment joints, stability and fatigue damage of structure should also be considered in the whole design due to repeated siltation and dredging.

Furthermore, the monitoring and sample simulation should also be conducted to verify the precision of the proposed analytical conclusions using FEM in the future.

\section{Acknowledgements}

This work was financially supported by the National high technology research and development program (863) (2006 aa11z119).

\section{References}

[1] Paul L.A. Erftemeijer, Bernhard Rieg, Bert W. Hoeksema, et al. Environmental impacts of dredging and other sediment disturbances on corals: A review. Marine Pollution Bulletin, Vol. 64 (2012): p. 1737-1765.

[2] Nestor S. Rasmussen. Dredging for Immersed tunnels: the Designer's and Contractor's Viewpoint. Central Dredging Association. CEDA Dredging Days 1987. ICE Publishing, p. 75-87.

[3] Walter C Grantz. Immersed tunnel settlements. Part 2: case histories. Tunnelling and Underground Space Technology, Vol. 16 (2001) p. 203-210.

[4] Walter C Grantz. Immersed tunnel settlements. Part 1: nature of settlements. Tunnelling and Underground Space Technology, Vol. 16 (2001) p. 195-201.

[5] Huang Jun , Zhang Dingli , Liu Hongzhou , et al. Study on Influences of Channel Dredging on Longitudinal Stability of Subsea Shield Tunnel. Chinese Journal of Rock Mechanics and Engineering , Vol. 26 (2007) p.2363- 2369 (in Chinese).

[6] Duc Thang Chu, Gen Himori, Yuki Saito, et al. Study of Beach Erosion and Evolution of Beach Profile Due to Nearshore Bar Sand Dredging[J]. Procedia Engineering, Vol. 116 (2015) p. 285-292.

[7] Wang Mengshu. Current Developments and Technical Issues of Underwater Traffic Tunnel-Discussion on Construction Scheme of Taiwan Strait Undersea Railway Tunnel. Chinese Journal of Rock Mechanics and Engineering, Vol. 27 (2008) p. 2161-2172 (in Chinese).

[8] Song Haoran, Zhang Dingli, Tan Guangzong. Study on risk assessment and control of Dalian bay subsea tunnel. Journal of Beijing Jiaotong University, Vol. 37 (2013) p. 1-6 (in Chinese).

[9] V. Magnenet, A. Giraud, F. Homand. Parameter sensitivity analysis for a Drucker-Prager model following from numerical simulations of indentation tests. Computational Materials Science, Vol. 44 p. 385-391.

[10] Y. Nakasone and S. Yoshimoto, T. A. Stolarski. Engineering analysis with ANSYS software. Elsevier/Butterworth-Heinemann, 2006. 